\title{
EP-121
}

\section{Empyema gall bladder in cirrhotic patient}

\author{
Muhammad ZAKRIA*
}

Department of Surgery, Wapda Teaching Hospital, Lahore, Pakistan

Introduction: Acute cholecystitis is initially managed by conservative management. Once patient is stable surgical treatment is the final solution. If stone is impacted neck of the gall bladder then we have to do surgery urgently as the conservative treatment may fail. Sometimes patient has late presentation and gall bladder is full of pus. In these conditions we have to select the surgery as our first option. This becomes more challenging if patient is cirrhotic and has other co morbid factors like diabetes mellitus. It is wise to do the immediate surgery in a cirrhotic patient or even non cirrhotic patient if he develops empyema gall bladder.

Methods: A 58-year-old female patient was admitted through emergency with complaint of pain right side abdomen and radiating to back. It was associated with fever, nausea, and vomiting and tenderness right side abdomen. She was a patient of child A, liver cirrhosis. She was diabetic but controlled on insulin. Her computed tomography scan was showing stone in the neck of gall bladder. She underwent open surgery through right Kocher incision, pus was aspirated from the gall bladder fundus. Retrograde cholecystectomy was done. Wound was left open. After 10 days secondary suturing was done.

Results: During her stay in hospital she remained afebrile postoperatively. Recovery was uneventf

Conclusions: Early intervention is gold standard in empyema gall bladder. Although laparoscopic cholecystectomy is very much ideal for the gall bladder surgery but in case of massive adhesions, open surgery is safe option if laparoscopic surgery fails. 